\title{
VisDa: creación de un plugin para WordPress
}

Autores: Cristina Fàbregas, Jordi Gervàs, Antonio Laiz, Ricardo Lodroño.

Coordinación: Laia Blasco, Quelic Berga, Carlos Casado, César Córcoles. 


\title{
VisDa: creación de un plugin para WordPress
}

\author{
Grupo de trabajo formado por: Cristina Fábregas, Jordi Gervàs, Antonio Laiz,
} Ricardo Lodroño.

\section{Introducción}

A continuación os transcribo un fragmento del vídeo promocional de la convocatoria del taller:

"Octubre 2011. Workshop de visualización de datos para el desarrollo de un plugin para Wordpress: Processing.js, Javascript, PHP, MySQL, HTML5, Canvas, Interactividad y Creatividad, ¿te lo vas a perder?"

Desde luego la respuesta a la pregunta que se nos planteaba era un rotundo NO. Dejando de lado la eficacia publicitaria del citado video parecía muy interesante poder trabajar con toda esta cantidad de tecnologías junto a un equipo multidisciplinar de profesionales de la multimedia.

Lo cierto es que el encuentro valió la pena, no solamente por el resultado conseguido, sino también por haber podido compartir experiencias y conocimientos con todos y cada uno de los participantes.

\section{Estructura y objetivos del Workshop}

La duración del Workshop fue de una semana (de sábado a sábado) empezando y terminando con una sesión presencial en la sede de la UOC de Rambla Catalunya, en Barcelona, y mediante el trabajo a distancia de los miembros del grupo entre semana.

El objetivo del mismo era el de crear un plugin para la revista Mosaic que permitiera acceder de manera visual a todos los contenidos de los diez años de vida de la revista. Debido a la variedad de perfiles entre los participantes se realizó una didáctica introducción a las tecnologías y conceptos necesarios para poder tener una visión global al problema que se nos iba a plantear.

Para ello, durante la sesión del primer día, el profesorado del Grau en Multimèdia nos había preparado cuatro sesiones, breves pero efectivas, con la información básica necesaria. A saber: 
a. Visualización de la información, por Laia Blasco.

b. Codificar en y para Wordpress (PHP), por César Córcoles.

c. La base de datos de Wordpress (MySQL), por Carlos Casado.

d. Processing y processing.js, por Quelic Berga.

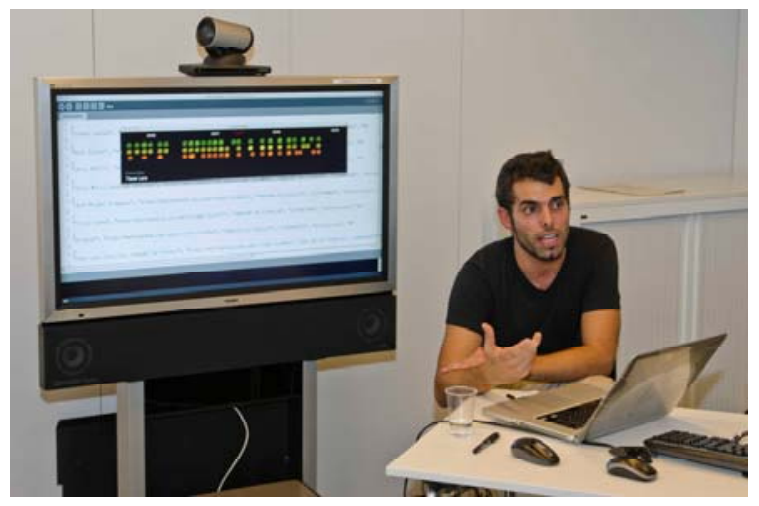

\section{Creación del plug-in}

\section{a. $1^{\text {a }}$ Sesión presencial (¿Y ahora qué?)}

i. Brainstorming: una vez sentadas las bases se empezó el proceso creativo mediante una 'lluvia de ideas' proceso que podría ser conocido también como "por dónde empezar cuando no tienes ni idea de por dónde". El objetivo, en este caso, era crear un boceto que sería el germen para el desarrollo del plugin.

ii. Los datos: una parte importante del proceso es saber cuál es la información que queremos mostrar al usuario y en qué momento: título del post, autor, contenido, fecha de publicación, visitas, tags,... etc. A nivel interno, a la entidad principal de información de Mosaic se le llama post. Ésta incluye artículos, entrevistas, recursos y experiencias.

iii. Bocetos: con un papel y un lápiz se pueden conseguir grandes diseños para responder a la pregunta ¿cómo mostrar esta información al usuario de manera efectiva e impactante?: espirales, esferas, círculos...etc.

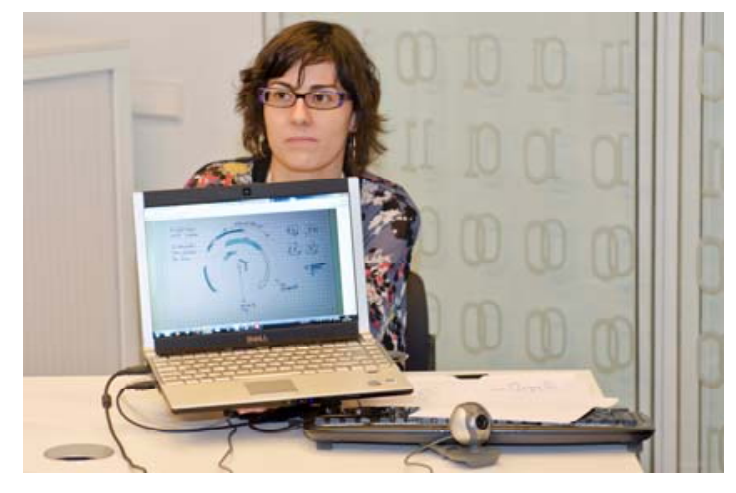




\section{b. Trabajo virtual BSCW (¡Démosle forma!)}

i. Estilo gráfico: una vez consensuados los datos que queremos mostrar al usuario se realizan una serie de propuestas visuales las cuales serán votadas democráticamente por los miembros del grupo. Aunque no nos engañemos, nadie había trabajado anteriormente con Processing por lo que elegimos una representación facilita, mediante figuras rectangulares, pero no se lo digáis a nadie.

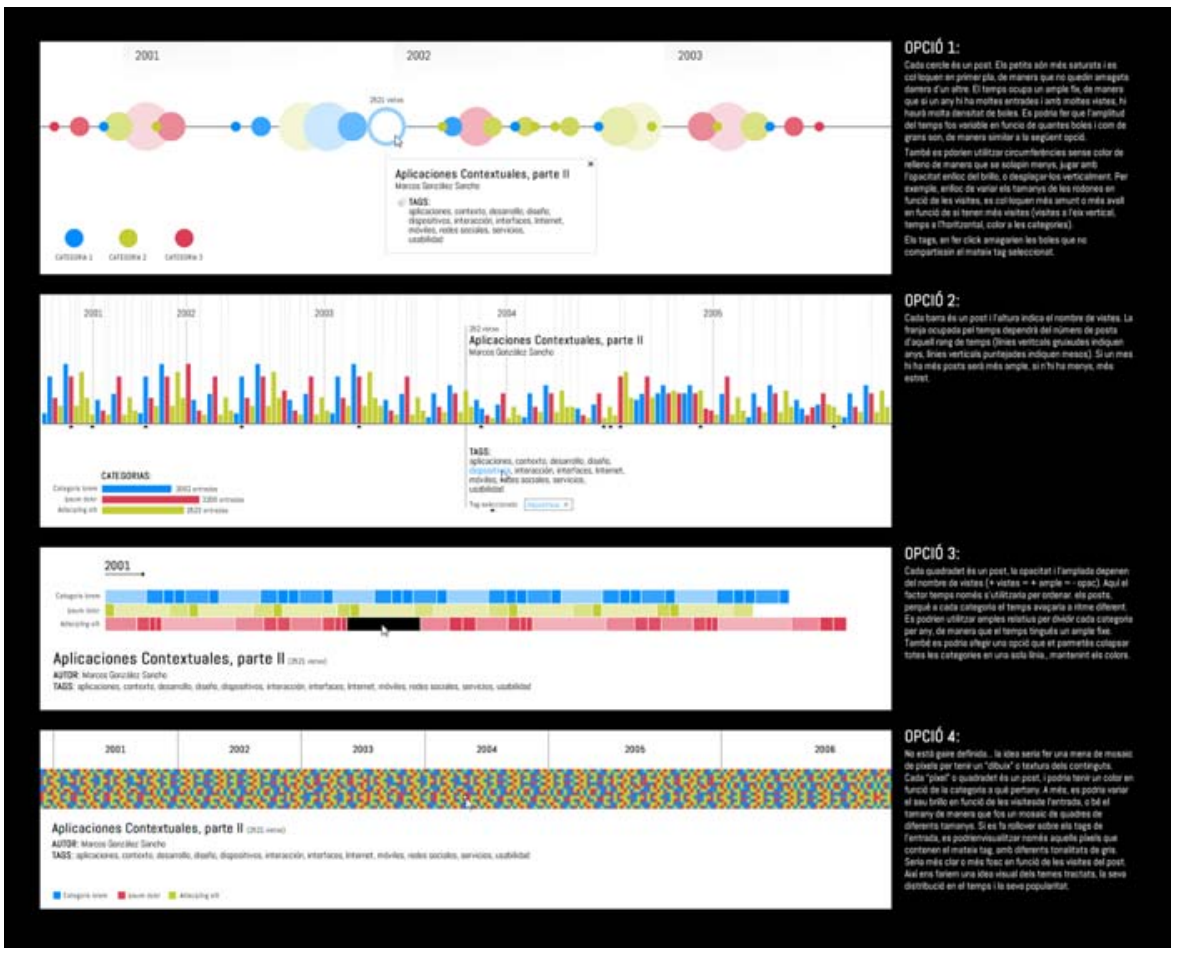

ii. Desarrollo del plug-in: partiendo del ejemplo proporcionado por el profesorado y utilizando la conocida técnica de ensayo y error se empezó a desarrollar la propuesta, siguiendo las directrices siguientes:

1. Metáfora del dial de radio: teníamos claro que el plugin debería permitir acceder al contenido íntegro de la revista, desde su creación hasta los artículos más novedosos, por lo que se creó una línea temporal que sería accesible mediante barrido del cursor.

2. Colores: las diferentes categorías presentes en Mosaic (entrevistas, artículos, experiencias y recursos) serían representadas cada una con un color diferente de manera que fueran visualmente identificables. 
3. Textos: en una primera pantalla se decidió mostrar: nombre de la categoría, año de publicación, título del post y número de visitas recibidas. Al hacer clic en ellas se accedería al contenido íntegro del post mediante el navegador web.

4. Tamaño de las barras (visitas): además de poder identificar la categoría a la que pertenece la información mostrada, se creyó interesante resaltar de alguna manera su relevancia tomando como indicador el número de visitas recibidas.

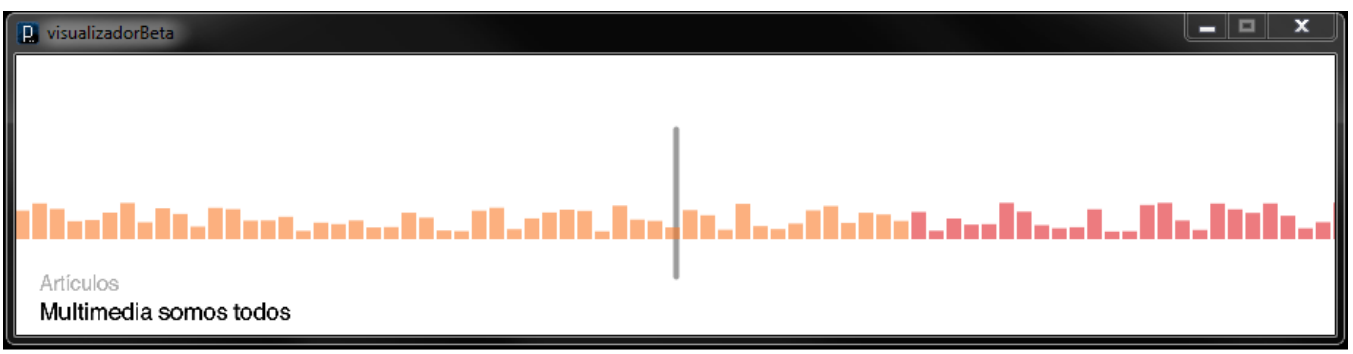

\section{c. 2a sesión presencial (Esto hay que arreglarlo)}

Durante la segunda sesión se realizó una puesta en común del trabajo realizado de manera virtual. El profesorado nos ayudó a acabar de solventar dudas y problemas.

i. Integración en el entorno de desarrollo: aunque el plugin se había desarrollado en Processing era necesario integrarlo en wordpress para poderlo ejecutar, conocer la estructura de su base de datos en mysql para extraer la información necesaria mediante sentencias sql y un poco de php para comprender cómo recuperar la información que serviría de datos de entrada al script de Processing.

ii. Espacio de Pruebas: se nos proporcionó un servidor de pruebas dedicado donde poder realizar cambios y testear lo desarrollado sin límites de ningún tipo. Dado que el desarrollo no estaba finalizado se decidió realizar los últimos cambios en los días posteriores.

\section{d. Trabajo virtual BSCW (últimos retoques)}

Antes de dar por finalizado el desarrollo del plugin se revisó todo el código antes de presentarlo públicamente en la celebración de los diez años de Mosaic.

\section{i. Decisiones de diseño}

1. Transparencia: para dotar de un poco más de dinamismo a lavisualización se utiliza la opacidad para diferenciar la información 
más novedosa (más opaca) de la más antigua (más transparente). Hay que tener en cuenta que el usuario sólo está viendo, en primera instancia, barras de colores, por lo que cuanta más información se le proporcione mejor.

2. Proporción de las alturas de las barras: teniendo en cuenta que el espacio disponible para incrustar el plugin en la web es finito necesitábamos una manera de acotar la altura de las barras en apenas 200 píxeles. Tomando como altura máxima el post con mayor número de visitas se realiza una proporción con el resto de elementos utilizando una escala logarítmica, de manera que simule las diferencias entre ellos en cuanto a números de visitas.

3. Ordenación de los datos: el cursor se posiciona, por defecto, en la parte derecha del plugin de manera que se visualicen primero los posts más recientes.

ii. Versión final: integración de todos los cambios realizados y testeo. Comentar que es posible que el plugin no funcione en navegadores antiguos.

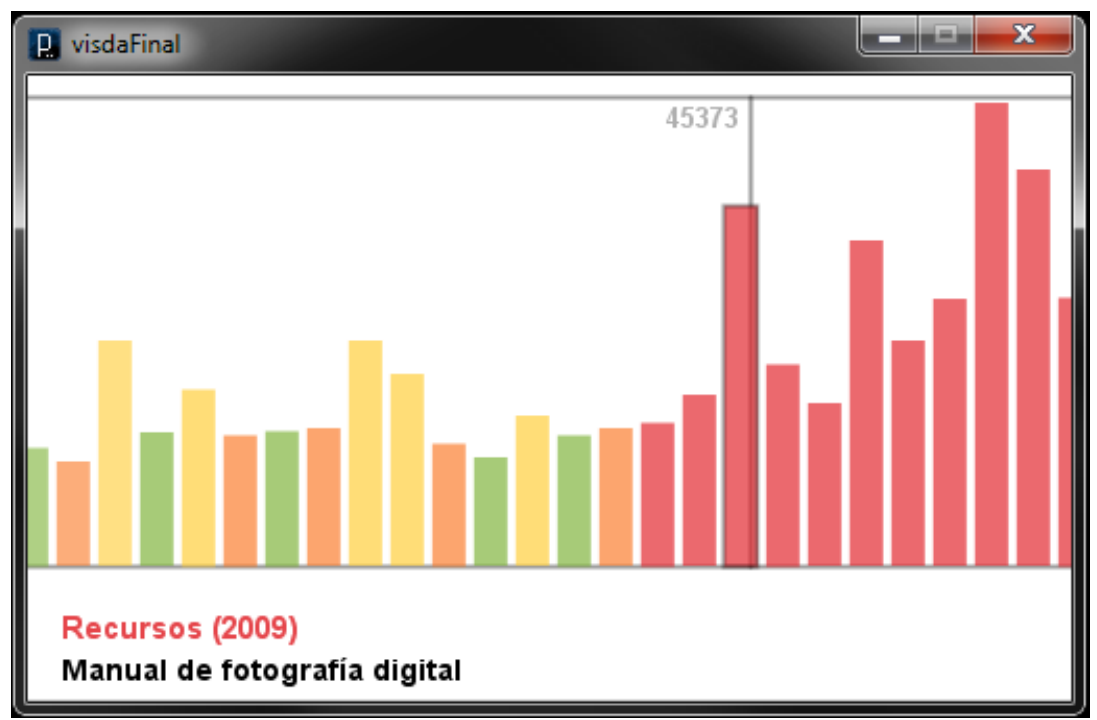

iii. Publicación: 'Processing.js' es una librería de Javascript que permite la ejecución dentro de un navegador de programas implementados con el lenguaje Processing y sin necesidad de tener que ejecutar un Applet de Java. Se encontraron algunas inconsistencias al hacer el paso de un sistema a otro, aunque no afectaron a la ejecución final. 


\section{Instalación}

Para instalar el Plugin

1. Descomprimir el archivo zip del plugin en la carpeta wp-content/plugins.

2. Activarlo desde el administrador de WordPress.

3. Poner el shortcode correspondiente entre claudátors dentro del post dónde se quiera visualizar [visda] en este caso.

\section{Conclusión}

En este tipo de actividades de corto recorrido hay que tener muy presente el tiempo disponible y las habilidades de cada uno por lo que se intentó plasmar un objetivo no demasiado ambicioso pero factible y parece ser que el resultado fue satisfactorio.

Podemos decir que una vez finalizado el Workshop se adquirió una visión general de la estructura de Mosaic dentro de Wordpress y pudimos conocer de primera mano las herramientas necesarias para poder realizar este tipo de desarrollos.

Este Workshop nos ha abierto un nuevo campo de trabajo relacionado con la visualización de la información que consideramos muy interesante y esperamos poder seguir desarrollando en un futuro.

Y por último, pero no por ello menos importante, debemos nombrar al lenguaje de programación 'Processing' pieza clave en este proceso y que desde aquí os emplazamos a probarlo y a disfrutarlo.

¿Cómo? Ah, ¿que no sabéis programar? ¡Pues es ideal para vosotros! Ya, pero ¿que no sabéis diseñar? ¡iPues es ideal para vosotros!! http://sketch.processing.org/ 